'Vicerrectoría de Investigación y Postgrado. Centro de Investigación en Estudios Avanzados del Maule, Universidad Católica del Maule. Talca, Chile

${ }^{2}$ Departamento de Ciencias del Deporte y Acondicionamiento Físico. Universidad Católica de la Santísima Concepción. Concepción, Chile. ${ }^{3}$ Institute of Health and Wellbeing, University of Glasgow. Glasgow, United Kingdom. 4Escuela de Kinesiología. Facultad de Salud. Universidad Santo Tomás. Chile. Universidad Tecnológica de Chile, INACAP, Chile.

${ }^{6}$ Instituto de Anatomía, Histología y Patología. Facultad de Medicina. Universidad Austral de Chile. Valdivia, Chile.

${ }^{7}$ Instituto de Farmacia, Facultad de Ciencias, Universidad Austral de Chile. Valdivia, Chile. ${ }^{8}$ CIEDE-UCSC, Facultad de Medicina, Universidad Católica de la Santísima Concepción. Concepción, Chile.

${ }^{9}$ Escuela de Educación Física, Universidad San Sebastián. Concepción, Chile.

${ }^{10} \mathrm{Grupo}$ de Investigación en Calidad de Vida, Departamento de Ciencias de la Educación,

Facultad de Educación y Humanidades, Universidad del Biobío. Chillán, Chile.

"Departamento de Educación Física, Facultad de Educación, Universidad de Concepción. Concepción, Chile.

${ }^{12}$ Centro de Vida Saludable, Universidad de Concepción. Concepción, Chile.

${ }^{13}$ Departamento de Ciencias de la Actividad

Física. Núcleo de Investigación en Salud,

Actividad Física y Deporte. Laboratorio de

Rendimiento Humano. Universidad de Los Lagos. Osorno, Chile.

${ }^{14}$ Grupo IRyS, Escuela de Educación Física, Pontificia Universidad Católica de Valparaíso. Valparaíso, Chile.

${ }^{15}$ Departamento de Pediatría y Cirugía Infantil,

Facultad de Medicina, Universidad de La Frontera. Temuco, Chile.

${ }^{16}$ Institute of Cardiovascular and Medical

Sciences, University of Glasgow. Glasgow, United Kingdom.

${ }^{7}$ Centro de Investigación en Fisiología de Ejercicio - CIFE, Universidad Mayor. Santiago, Chile.

aProfesor Educación Física.

${ }^{\mathrm{b}}$ Nutricionista.

'Kinesióloga.

dBioquímica.

*JVG y CMC contribuyeron igualmente a este trabajo y son considerados primer autor compartido

Trabajo no recibió financiamiento. Los autores declaran no tener conflictos de interés.

Recibido el 28 de junio de 2018, aceptado el 22 de agosto de 2019.

Correspondencia a:

Dr. Carlos Celis-Morales Glasgow Cardiovascular Research Centre Institute of Cardiovascular and Medical Science University of Glasgow. G12 8TA, Glasgow. United Kingdom. Carlos.Celis@glasgow.ac.uk

\section{Caracterización de los estilos de vida en dueñas de casa chilenas. Análisis de la Encuesta Nacional de Salud 2009-2010}

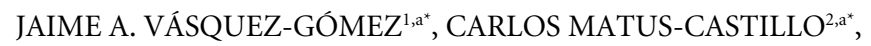
FANNY PETERMANN-ROCHA ${ }^{3, \mathrm{~b}}$, YENY CONCHA-CISTERNAS ${ }^{4,5, \mathrm{c}}$, ANA MARÍA LEIVA ${ }^{6, d}$, MARÍA ADELA MARTÍNEZ-SANGUINETTI ${ }^{7, d}$, CLAUDIA TRONCOSO-PANTOJA ${ }^{8, \mathrm{~b}}$, ALEX GARRIDO-MENDEZ ${ }^{2,9, \mathrm{a}}$, XIMENA DÍAZ-MARTÍNEZ ${ }^{10, a}$, CARLOS SALAS ${ }^{11, a}$, NATALIA ULLOA ${ }^{12, \mathrm{~d}}$, CRISTIAN ÁLVAREZ ${ }^{13, \mathrm{a}}$, RODRIGO RAMÍREZ-CAMPILLO ${ }^{13, a}$,

FERNANDO RODRÍGUEZ-RODRÍGUEZ ${ }^{14, a}$, CARLOS CRISTI-MONTERO ${ }^{14, a}$, FABIÁN LANUZA ${ }^{15, b}$, CARLOS CELIS-MORALES ${ }^{16,17, a}$, en representación del Grupo de Investigación ELHOC

\section{Lifestyles of Chilean housewives. Analysis of the 2009-2010 Chilean Health Survey}

Background: Housewives represent a important proportion of the Chilean population. However, there is limited evidence about their lifestyles. Aim: To characterize lifestyles and determine the level of compliance with healthy lifestyles guidelines of housewives in Chile. Material and Methods: Housewives from the 2009-2010 National Health Survey were included. The variables studied included levels of physical activity (PA), sedentary behavior, diet, hours of sleep and smoking. Compliance with healthy lifestyle behaviors was evaluated through logistic regression, granting a value of 1 for compliance and 0 for non-compliance. A healthy lifestyle was defined as meeting at least four healthy behaviors. Results: Housewives aged $>55$ years had a higher BMI and waist circumference compared to those aged $<40$ years. Housewives were also more likely to report moderate alcohol consumption and were more likely to meet a healthier lifestyle score (Odds ratio $=1.52$ [95\% confidence intervals: 1.09 to 2.11], $p=0.013)$. No significant age trends were observed for other lifestyle behaviors. Conclusions: Housewives had high levels of central obesity, excess body weight and high levels of salt intake but low alcohol intake. Their healthy lifestyles behaviors increased along with increasing age.

(Rev Med Chile 2019; 147: 1144-1153)

Key words: Exercise; Diet; Life Style; Obesity; Women. 
O hile ha enfrentado un conjunto de cambios sociodemográficos y en la estructura familiar en los últimos años. La creciente incorporación de las mujeres al mercado laboral ha conllevado a que tanto ellas como los hombres deban compatibilizar roles, tanto en lo público como en lo privado ${ }^{1}$. No obstante, y a pesar de que desde el año 1990 se ha visto un incremento de hogares monoparentales encabezados en su mayoría por mujeres jefas de hogar y sostenedoras económicas ${ }^{2}$, las mujeres aún continúan siendo las principales responsables de las labores y quehaceres del hogar. El trabajo doméstico, cuyo objetivo es la producción de bienes y servicios para satisfacer las necesidades de los miembros del hogar ${ }^{3}$, es conocido coloquialmente en Chile como una labor a realizar por amas o dueñas de casa.

En Chile, $24,7 \%$ de la población declara no ser activa laboralmente (porcentaje que incluye las labores domésticas no remuneradas), de esta cifra, 97,3\% son mujeres ${ }^{4}$. La baja participación de las mujeres en la actividad económica productiva es una característica de los países en vías de desarrollo ${ }^{5}$, en los cuales se encuentra Chile ${ }^{6}$. Esta población, junto a personas de menor nivel educacional y desempleados, presentan un menor conocimiento sobre enfermedades asociadas a estilos de vida no saludables ${ }^{7,8}$, lo que podría gatillar un aumento del riesgo cardiovascular asociado a bajos niveles de actividad física (AF) y a una ingesta energética excesiva por un período prolongado ${ }^{9}$. En este contexto, y considerando que existe un limitado número de estudios sobre estilos de vida en este grupo sociodemográfico ${ }^{9,10}$, el objetivo de este trabajo fue caracterizar los estilos de vida y determinar el nivel de cumplimiento de recomendaciones de estilos de vida saludable en dueñas de casa chilenas.

\section{Materiales y Métodos}

\section{Diseño del estudio}

La muestra seleccionada corresponde a las dueñas de casa que formaron parte de la Encuesta Nacional de Salud de Chile 2009-2010 (ENS 20092010) ${ }^{11}$. La ENS 2009-2010 es una encuesta de prevalencia, realizada en hogares, con una muestra nacional, probabilística, estratificada y multietápica, con representatividad nacional, regional, y área urbana/rural, que incluyó a 5.412 personas. Sin embargo, solo 1.223 mujeres entre 18 y 94 años, que reportaron ser dueñas de casa, fueron incluidas en este estudio. Al aplicar el factor de expansión sugerido por la ENS 2009-2010, la muestra expandida correspondió a 2.455.059 dueñas de casa. El estudio se restringió solamente a mujeres ya que solo 33 hombres reportaron realizar esta labor. El protocolo de la ENS 2009-2010 fue aprobado por el Comité de Ética del Departamento de Medicina de la Pontificia Universidad Católica de Chile, y todos los participantes firmaron un consentimiento informado ${ }^{11}$.

\section{Clasificación de las dueñas de casa}

Se clasificó como dueña o ama de casa a todas las mujeres que administraban su hogar como principal ocupación ${ }^{10}$. Para evaluar diferencias entre los distintos patrones y estilos de vida, se subdividió a las dueñas de casa en 3 grupos de edad: $<40$ años; 40-55 años; $>55$ años.

\section{Mediciones antropométricas}

El estado nutricional fue determinado en base al índice de masa corporal $\left(\mathrm{IMC} \mathrm{kg} / \mathrm{m}^{2}\right)$ : bajo peso: $<18,5 \mathrm{~kg} / \mathrm{m}^{2}$; normo peso: $18,5 \mathrm{a} 24,9 \mathrm{~kg} / \mathrm{m}^{2}$; sobrepeso: $25,0-29,9 \mathrm{~kg} / \mathrm{m}^{2}$ y obesidad: $\geq 30,0 \mathrm{~kg} /$ $\mathrm{m}^{2}$ (incluye obesidad mórbida) ${ }^{12}$, y para adultos mayores se utilizó la siguiente clasificación: bajo peso: $<22,9 \mathrm{~kg} / \mathrm{m}^{2}$; normo peso: 23,0 a $27,9 \mathrm{~kg} /$ $\mathrm{m}^{2}$; sobrepeso: 28,0 a $31,9 \mathrm{~kg} / \mathrm{m}^{2}$ y obesidad $\geq 32,0$ $\mathrm{kg} / \mathrm{m}^{2}{ }^{13}$. La obesidad central fue definida como un perímetro de cintura $(\mathrm{PC}) \geq 88 \mathrm{~cm}$ para mujeres ${ }^{11}$.

\section{Variables sociodemográficas y de estilo de vida}

Las variables sociodemográficas (edad, sexo, nivel educacional, ingreso económico, zona geográfica) y de estilo de vida, como el tabaquismo, autorreporte de salud y bienestar, consumo de alcohol, consumo de sal (obtenido a través de muestra de orina y transformado en sodio calculado por la ecuación de regresión de Tanaka et al. 2002), y consumo de frutas y verduras se obtuvieron mediante la aplicación de cuestionarios validados en la ENS 2009-2010 ${ }^{11}$.

Los niveles de AF, incluyendo el tiempo destinado a AF asociada al transporte, AF de intensidad moderada y vigorosa fueron estimados con el cuestionario "Global Physical Activity Questionnaire"14. La AF total es la suma del tiempo en actividades 
de transporte, de intensidad moderada y vigorosa, en el trabajo y en el tiempo libre, siendo expresada en equivalentes metabólicos (MET) por horas y semana. El punto de corte para inactividad física fue un gasto energético menor a $600 \mathrm{MET} / \mathrm{min} /$ semana ( $>150$ min de AF a la semana). También se estimó el tiempo destinado a AF moderada y vigorosa, el cual fue expresado como MET/h/ semana para tomar en cuenta las diferencias en gasto energético asociado a ambas intensidades de AF. Los niveles de sedentarismo fueron estimados mediante el mismo cuestionario a partir del autorreporte de tiempo destinado a actividades que involucren estar sentado o reclinado durante el tiempo libre o de trabajo. Se consideró alto nivel de sedentarismo cuando una persona destinaba más de 4 h a estar sentada al día (correspondiente a la mediana de la población $)^{15}$.

\section{Índice de estilo de vida saludable}

Se utilizaron 7 conductas relacionadas a un estilo de vida saludable: 1 ) consumo de 5 porciones de frutas y verduras al día; 2) consumo de sal $<8 \mathrm{~g} /$ día; 3$)$ consumo no riesgoso de alcohol $(<8$ puntos test de identificación de trastornos debido al consumo de alcohol [AUDIT] ) ${ }^{16} ; 4$ ) horas de sueño entre 7-9 h al día; 5) nunca haber fumado; 6) ser físicamente activo ( $>150 \mathrm{~min}$ de $\mathrm{AF}$ a la semana); y 7) tiempo sedente $<4 \mathrm{~h}$ al día ${ }^{15}$. A los individuos que cumplían con estas recomendaciones se les asignaba 1 punto por cada conducta saludable que cumplían, mientras que aquellos que no cumplían con estas recomendaciones se les asignaban un valor cero. El puntaje máximo fue de 7 puntos, lo que indicaba que se cumplían todas las conductas, siendo el mínimo 0 , que representaba no cumplir ninguna recomendación. Para fines de este estudio, se clasificó a los participantes con un estilo de vida saludable si presentaban un puntaje $\geq 4$, lo cual representa un cumplimiento de $57 \%$ de las 7 conductas saludables incluidas en este estudio. Por ende, aquellas dueñas de casa que reportaron $\leq 3$ conductas fueron definidas como estilo de vida no saludable. Este punto de corte para definir un estilo de vida saludable fue definido por los investigadores de este estudio y no basada en una escala validada.

\section{Análisis estadístico}

Todos los análisis fueron realizados con STATA (versión MP 15.0) y el comando de "svyset" para muestras complejas (https://www.stata.com/ manuals13/svy.pdf) y tomando en consideración los factores de expansión sugeridos por la ENS 2009-2010.

Las características de la población son presentadas como promedios para variables continuas y como porcentaje para variables categóricas, con sus respectivos $95 \%$ de intervalo de confianza (95\%IC). Estos datos son presentados para la población total de "dueña o ama de casa" y también por grupos etarios (<40, de 40 a 55 y $>55$ años). No se realizaron comparaciones estadísticas entre los grupos (Tabla 1), como lo sugieren las recomendaciones para estudios de corte transversal STROBE, ya que dichas comparaciones podrían aumentar la probabilidad de un error estadístico tipo 1.

Para identificar si el promedio de las variables de salida (adiposidad, estilos de vida, alimentación, AF, tiempo sedente) presentan una tendencia significativa a disminuir o aumentar según grupo etario, se realizaron análisis de regresión lineal para muestras complejas y corregidos por el factor de expansión de la ENS. La variable edad fue introducida en los modelos estadísticos como variable ordinal $(0=$ grupo $<40$ años, $1=40-55$ años, $y$ $2=$ a personas $>55$ años). Por ende, el valor $\mathrm{p}$ de tendencia indica si se observa una disminución o aumento de las variables de resultado a mayor edad y tomando como referencia al grupo etario más joven ( $<40$ años).

Para evaluar el cumplimiento de las recomendaciones de las conductas saludables entre los grupos, se realizó análisis de regresión logística corregidos por los factores de expansión, donde la variable de salida fue codificada de forma binaria $(0=$ no cumple con la recomendación para una conducta saludable y $1=$ cumple con la recomendación), mientras que los grupos etarios fueron introducidos en el modelo estadístico como variable ordinal $(0=<40$ años, $1=40-55$ años y 2 $=>55$ años), asignando como referencia al grupo $<40$ años. Los resultados de estos análisis fueron reportados como odds ratio (OR) y sus respectivos 95\% IC. Por ende, un OR $<1$ indicaría que no cumplen con la recomendación de estilo de vida saludable y un OR $>1$ indicaría que cumplen con la recomendación o conducta saludable.

Todos los análisis fueron ajustados por las variables de confusión, nivel educacional y zona de residencia (rural, urbana). El nivel de significación estadística fue definido como $\mathrm{p}<0,05$. 
Tabla 1. Características de la población de dueñas de casa en Chile según edad

\begin{tabular}{|c|c|c|c|c|}
\hline \multirow{2}{*}{$\begin{array}{l}\text { Características } \\
\text { socio-demográficas }\end{array}$} & \multirow[b]{2}{*}{ Total } & \multicolumn{3}{|c|}{ Grupo etario (años) } \\
\hline & & $<40$ & 40-55 & $>55$ \\
\hline n expandido $*$ & 2.455 .059 & 876.003 & 904.800 & 674.256 \\
\hline Edad (años)* & $46,5(42,4 ; 50,6)$ & $33,9(31,2 ; 36,7)$ & $47,2(45,4 ; 49,0)$ & $65,0(61,3 ; 68,7)$ \\
\hline \multicolumn{5}{|l|}{ Zona geográfica, (\%) } \\
\hline Rural & $20,5(17,5 ; 23,8)$ & $26,4(20,2 ; 33,5)$ & $16,2(12,2 ; 21,2)$ & $18,6(14,0 ; 24,2)$ \\
\hline Urbano & $79,5(76,2 ; 82,5)$ & $73,6(66,4 ; 79,8)$ & $83,8(78,8 ; 87,8)$ & $81,3(75,7 ; 85,9)$ \\
\hline Años de educación* & $11,2(10,1 ; 12,3)$ & $10,8(9,8 ; 11,9)$ & $13,1(11,1 ; 15,2)$ & $9,2(7,6 ; 10,8)$ \\
\hline \multicolumn{5}{|l|}{ Nivel educacional, (\%) } \\
\hline Bajo (< 8 años) & $27,1(23,3 ; 31,3)$ & $11,1(7,6 ; 16,1)$ & $25,2(18,6 ; 33,2)$ & $50,4(42,3 ; 58,4)$ \\
\hline Media (8 a 12 años) & $63,9(59,3 ; 68,3)$ & $76,4(69,5 ; 82,1)$ & $66,8(58,4 ; 74,2)$ & $43,7(35,9 ; 51,9)$ \\
\hline Alto (>12 años) & $9,0(6,6 ; 12,1)$ & $12,4(8,2 ; 18,4)$ & $8,0(4,7 ; 13,5)$ & $5,8(2,6 ; 12,5)$ \\
\hline \multicolumn{5}{|l|}{ Ingreso económico, (\%) } \\
\hline Bajo (< 250 mil pesos) & $63,6(58,7 ; 68,1)$ & $64,3(56,1 ; 71,7)$ & $63,8(55,1 ; 71,7)$ & $62,3(53,9 ; 70,0)$ \\
\hline Medio (250 a 450 mil pesos) & $31,3(26,9 ; 36,1)$ & $31,8(24,6 ; 40,0)$ & $30,9(23,4 ; 39,5)$ & $31,3(24,0 ; 39,7)$ \\
\hline Alto (> 450 mil pesos) & $5,1(3,6 ; 7,2)$ & $3,9(2,1 ; 6,9)$ & $5,3(3,2 ; 8,8)$ & $6,4(3,1 ; 12,6)$ \\
\hline \multicolumn{5}{|l|}{ Características antropométricas } \\
\hline Estatura $(m)^{*}$ & $1,6(1,5 ; 1,6)$ & $1,5(1,5 ; 1,6)$ & $1,6(1,5 ; 1,6)$ & $1,6(1,5 ; 1,6)$ \\
\hline Peso corporal $(\mathrm{kg})^{*}$ & $65,6(61,7 ; 69,7)$ & $66,0(57,8 ; 74,3)$ & $64,9(60,6 ; 69,1)$ & $66,3(59,5 ; 73,1)$ \\
\hline $\mathrm{IMC}\left(\mathrm{kg} / \mathrm{m}^{2}\right)^{*}$ & $27,1(25,7 ; 28,6)$ & $28,0(25,1 ; 30,9)$ & $26,4(24,7 ; 28,1)$ & $26,7(24,0 ; 29,4)$ \\
\hline \multicolumn{5}{|l|}{ Estado nutricional, (\%) } \\
\hline Bajo peso & $1,3(0,6 ; 2,7)$ & $1,2(0,5 ; 2,9)$ & $0,8(0,1 ; 5,0)$ & $2,0(0,6 ; 6,9)$ \\
\hline Normal & $28,0(23,5 ; 33,0)$ & $44,1(35,6 ; 52,9)$ & $23,1(16,6 ; 31,1)$ & $14,0(9,2 ; 20,7)$ \\
\hline Sobrepeso & $33,1(28,8 ; 37,6)$ & $25,5(19,6 ; 32,6)$ & $37,2(29,5 ; 45,7)$ & $37,2(29,8 ; 45,1)$ \\
\hline Obeso & $37,6(32,9 ; 42,5)$ & $29,1(22,6 ; 36,7)$ & $38,9(30,4 ; 48,1)$ & $46,8(38,9 ; 54,9)$ \\
\hline Perímetro de cintura $(\mathrm{cm})^{*}$ & $86,4(82,8 ; 90,0)$ & $86,6(80,0 ; 93,2)$ & $84,0(80,4 ; 87,6)$ & $89,1(81,7 ; 96,5)$ \\
\hline \multicolumn{5}{|l|}{ Obesidad central, (\%) } \\
\hline Normal & $77,8(73,7 ; 81,4)$ & $86,0(80,7 ; 90,0)$ & $78,0(70,1 ; 84,2)$ & $66,9(58,9 ; 74,0)$ \\
\hline Obeso & $22,2(18,6 ; 26,3)$ & $14,0(10,0 ; 19,3)$ & $22,0(15,8 ; 29,9)$ & $33,1(26,0 ; 41,1)$ \\
\hline \multicolumn{5}{|l|}{ Estilos de vida } \\
\hline Actividad física total (MET/hora/semana)* & $101,3(76,7 ; 125,9)$ & $108,1(62,2 ; 154,0)$ & $93,3(48,2 ; 138,4)$ & $101,3(62,0 ; 140,7)$ \\
\hline Actividad física de transporte (min/día)* & $41,6(25,7 ; 57,7)$ & $25,2(0,5 ; 49,9)$ & $60,6(38,6 ; 82,6)$ & $42,2(24,6 ; 59,9)$ \\
\hline Actividad física moderada (min/día)* & $109,1(79,1 ; 139,1)$ & $128,7(83,3 ; 174,0)$ & $82,6(28,9 ; 136,4)$ & $113,5(67,2 ; 159,9)$ \\
\hline Actividad física vigorosa (min/día)* & $33,2(12,3 ; 54,0)$ & $38,8(5,8 ; 83,5)$ & $28,3(1,3 ; 55,4)$ & $30,7(0,5 ; 60,9)$ \\
\hline MVPA (MET/hora/semana)* & $81,9(59,1 ; 104,6)$ & $96,3(54,5 ; 138,0)$ & $65,0(22,2 ; 107,8)$ & $81,6(45,9 ; 117,3)$ \\
\hline Inactivos < $600 \mathrm{MET} / \mathrm{min} /$ semana, (\%) & $22,3(19,1 ; 25,9)$ & $20,5(15,5 ; 26,7)$ & $17,7(13,1 ; 23,6)$ & $30,5(23,8 ; 38,2)$ \\
\hline Tiempo sedente (h/día)* & $2,7(2,3 ; 3,0)$ & $2,6(2,1 ; 3,1)$ & $2,9(2,0 ; 3,8)$ & $2,5(2,1 ; 2,8)$ \\
\hline Horas de sueño (h/día)* & $8,0(7,1 ; 8,9)$ & $8,5(6,7 ; 10,4)$ & $7,5(6,5 ; 8,6)$ & $7,8(6,7 ; 8,9)$ \\
\hline Consumo de frutas y verduras (g/día)* & $222,0(178,5 ; 265,4)$ & $160,0(118,6 ; 201,4)$ & $280,4(201,4 ; 359,4)$ & $241,2(193,4 ; 289,1)$ \\
\hline Consumo de sal (g/día)* & $8,3(7,6 ; 9,0)$ & $8,3(6,7 ; 9,9)$ & $8,0(7,2 ; 8,7)$ & $8,8(6,7 ; 8,9)$ \\
\hline Consumo de alcohol (puntos AUDIT)* & $1,38(1,15 ; 1,61)$ & $1,83(1,29 ; 2,36)$ & $1,22(0,94 ; 1,50)$ & $1,02(0,78 ; 1,25)$ \\
\hline \multicolumn{5}{|l|}{ Tabaquismo, (\%) } \\
\hline Nunca & $42,9(38,2 ; 47,6)$ & $38,6(31,1 ; 46,6)$ & $38,9(31,2 ; 47,3)$ & $53,7(45,6 ; 61,6)$ \\
\hline Ex-fumador & $20,6(16,6 ; 25,2)$ & $15,1(10,9 ; 20,5)$ & $25,2(17,2 ; 35,4)$ & $21,5(15,7 ; 28,7)$ \\
\hline Fumador & $36,6(31,9 ; 41,6)$ & $46,4(38,0 ; 54,9)$ & $35,8(28,1 ; 44,3)$ & $24,8(18,2 ; 32,8)$ \\
\hline \multicolumn{5}{|l|}{ Auto reporte de bienestar, (\%) } \\
\hline Mal & $4,1(2,7 ; 6,2)$ & $0,7(0,3 ; 1,6)$ & $6,2(3,7 ; 10,2)$ & $5,6(2,5 ; 12,0)$ \\
\hline Regular & $39,3(34,4 ; 44,3)$ & $36,8(28,7 ; 45,1)$ & $36,0(27,7 ; 45,1)$ & $46,8(38,9 ; 54,9)$ \\
\hline Buena & $56,7(51,7 ; 61,6)$ & $62,5(53,6 ; 70,6)$ & $57,8(48,9 ; 66,3)$ & $47,6(39,7 ; 55,5)$ \\
\hline
\end{tabular}

Datos presentados como media y $95 \%$ IC para variables continuas $\left.{ }^{*}\right)$. Variables categóricas son presentadas como \% y sus respectivos 95\% IC; ${ }^{¥}$ Presentan el número de personas incluidas en los análisis al aplicar el factor de expansión. IMC: índice de masa corporal; g: gramos; min: minuto; MET: metabolic equivalent of task; MVPA: Moderate to vigorous physical activity; AUDIT: Test de Identificación de trastornos debido al consumo de alcohol. 


\section{Resultados}

Las características generales de las dueñas de casa y su caracterización acorde a la edad son presentadas en la Tabla 1. En general, la población que reportó ser dueñas de casa poseía una edad promedio de 46,5 años (desviación estándar $[\mathrm{DE}]=15,2)$, un alto porcentaje de dueñas de casa habitaba en áreas urbanas (79,5\%), 63,9\% reportó un nivel de educación medio (8 a 12 años), mientras que $63,6 \%$ reportó estar en el tercil más bajo de ingreso económico familiar. Con relación al estado nutricional, 70,7\% de las dueñas de casa presentó un IMC $>25,0 \mathrm{~kg} / \mathrm{m}^{2}$, mientras que $22,2 \%$ presentó obesidad abdominal. En relación con la AF, 22,3\% reportó ser físicamente inactiva y en promedio reportaron destinar 2,7 $(\mathrm{DE}=2,3)$ horas al día en actividades de tipo sedente. En relación con otros estilos de vida, el consumo de sal ( $<8 \mathrm{~g} /$ día) y alcohol ( $<8$ puntos AUDIT) superó las recomendaciones internacionales, $42,9 \%$ se declaró no fumador y más de $55 \%$ declaró sentirse bien respecto al bienestar y salud.

En cuanto a variables antropométricas, el grupo $>55$ años presentaba en promedio un IMC y perímetro de cintura más alto en comparación a los grupos etarios más jóvenes (Figura 1). Para los niveles de AF y tiempo sedente, solo se encontraron diferencias significativas para AF de intensidad moderada, existiendo una tendencia significativa a realizar menos AF moderada en la medida que aumentaba la edad. Sin embargo, no se encontraron diferencias para otras intensidades de AF o tiempo sedente. Mientras que las horas de sueño reportadas en promedio fueron menores en los grupos de mayor edad en comparación a los grupos más jóvenes (Figura 2). En relación con el consumo de frutas, verduras, alcohol y sal en dueñas de casa según grupo etario, se observó que el consumo de frutas y verduras era mayor en el grupo de 40-55 años, mientras que el consumo de alcohol disminuía en la medida que incrementaba la edad (Figura 3 ).

Por último, al determinar el cumplimiento de las recomendaciones para un estilo de vida saludable (Figura 4), se observó una mayor asociación de cumplir con un consumo de alcohol no riesgoso ( $<8$ puntos AUDIT) en el grupo etario $>55$ años (OR = 2,45 [95\% IC: 1,14 a 5,25], $\mathrm{p}=$ $0,021)$ que en el grupo $<40$ años. Finalmente, es más probable que las dueñas de casa mayores a
55 años cumplieran con al menos 4 de un total de 7 estilos de vida saludable en comparación al grupo $<40$ años $(\mathrm{OR}=1,52$ [95\% IC: 1,09 a $2,11], \mathrm{p}=0,013)$. Todos estos resultados fueron independientes del nivel educacional y de la zona geográfica de residencia.
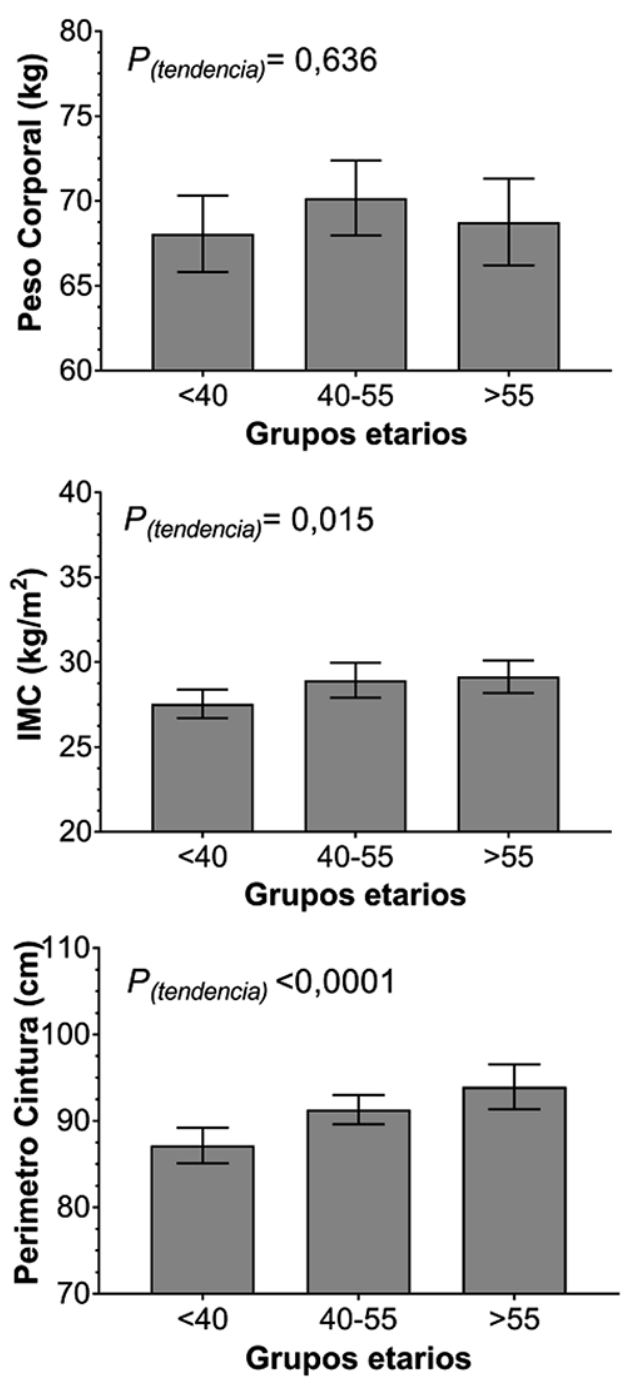

Figura 1. Niveles de adiposidad en dueñas de casa según grupo etario. Datos presentados como promedio y sus respectivos $95 \%$ IC, ajustados por nivel educacional y zona geográfica de residencia. El valor P-tendencia indica si el promedio de la variable de salida (IMC, perímetro de cintura, peso corporal) presenta una tendencia a ser menor o mayor en la medida que la edad aumenta. 

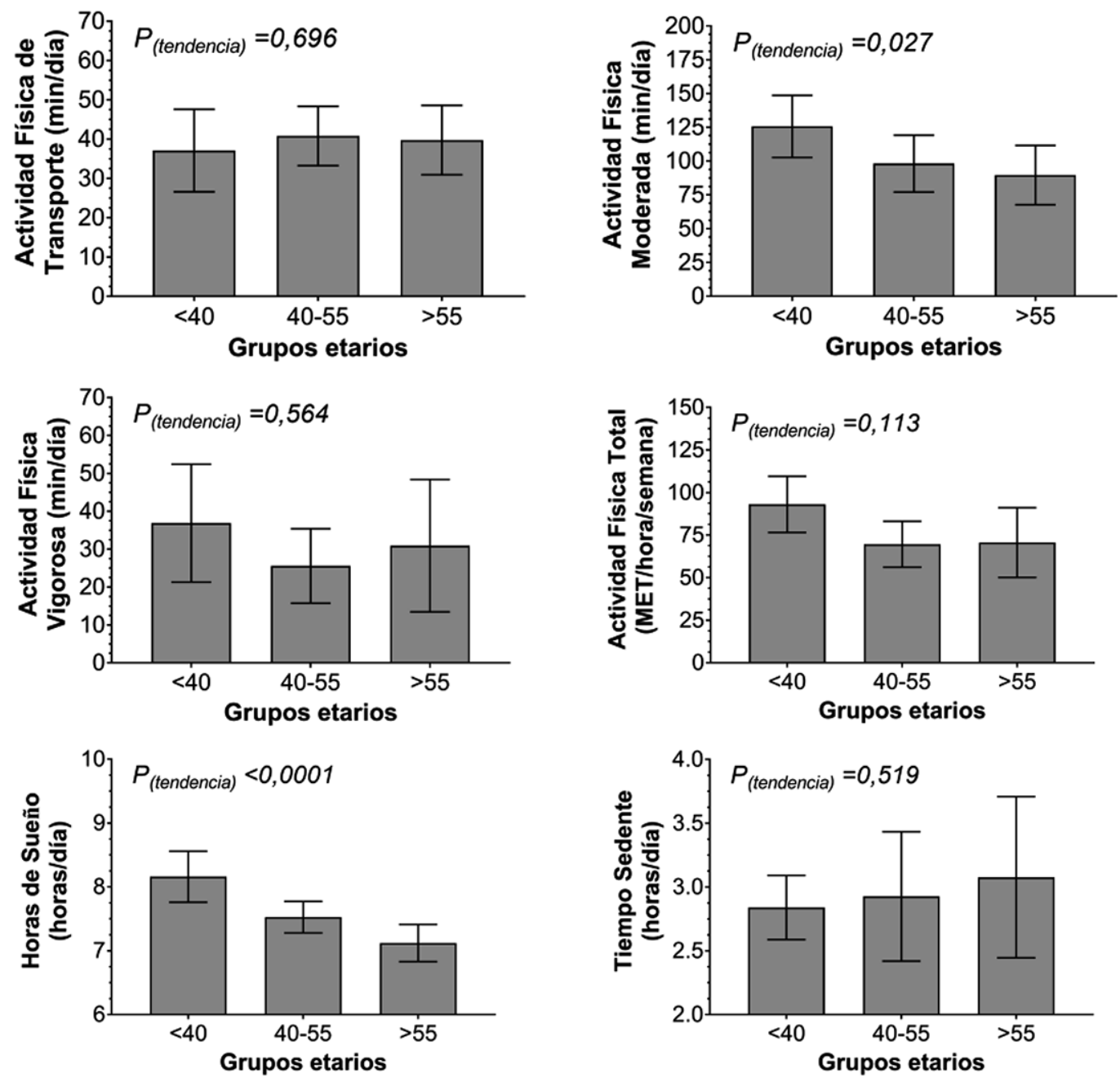

Figura 2. Niveles de actividad física, tiempo sedente y horas de sueño en dueñas de casa según grupo etario. Datos presentados como promedio y sus respectivos $95 \% \mathrm{IC}$, ajustados por nivel educacional y zona geográfica de residencia. El valor P-tendencia indica si el promedio de la variable de salida (actividad física, tiempo sedente u horas de sueño) presenta una tendencia a ser menor o mayor en la medida que la edad aumenta.

\section{Discusión}

En estudios poblacionales de otros contextos, los desempleados/dueñas de casa alcanzan a $41,7 \%$ de la población, siendo mujeres $68,8 \%$ de este segmento ${ }^{17}$ y dueñas de casa $62,2 \%{ }^{18}$. En Latinoamérica, las mujeres han comenzado a ocupar un rol importante en el ámbito laboral y se han alejado del rol de dueñas de casa ${ }^{19}$, lo que se aprecia en Chile ya desde la década 2000-0920,21 con una disminución del porcentaje de dueñas de casa en los últimos años ${ }^{22,23}$.

El principal hallazgo de este estudio demostró una mayor asociación en las dueñas de casa mayores a 55 años de cumplir las recomendaciones de estilos de vida saludable en comparación al grupo más joven ( $<40$ años). Principalmente, aquellas relacionadas con el consumo de alcohol y tabaco; 


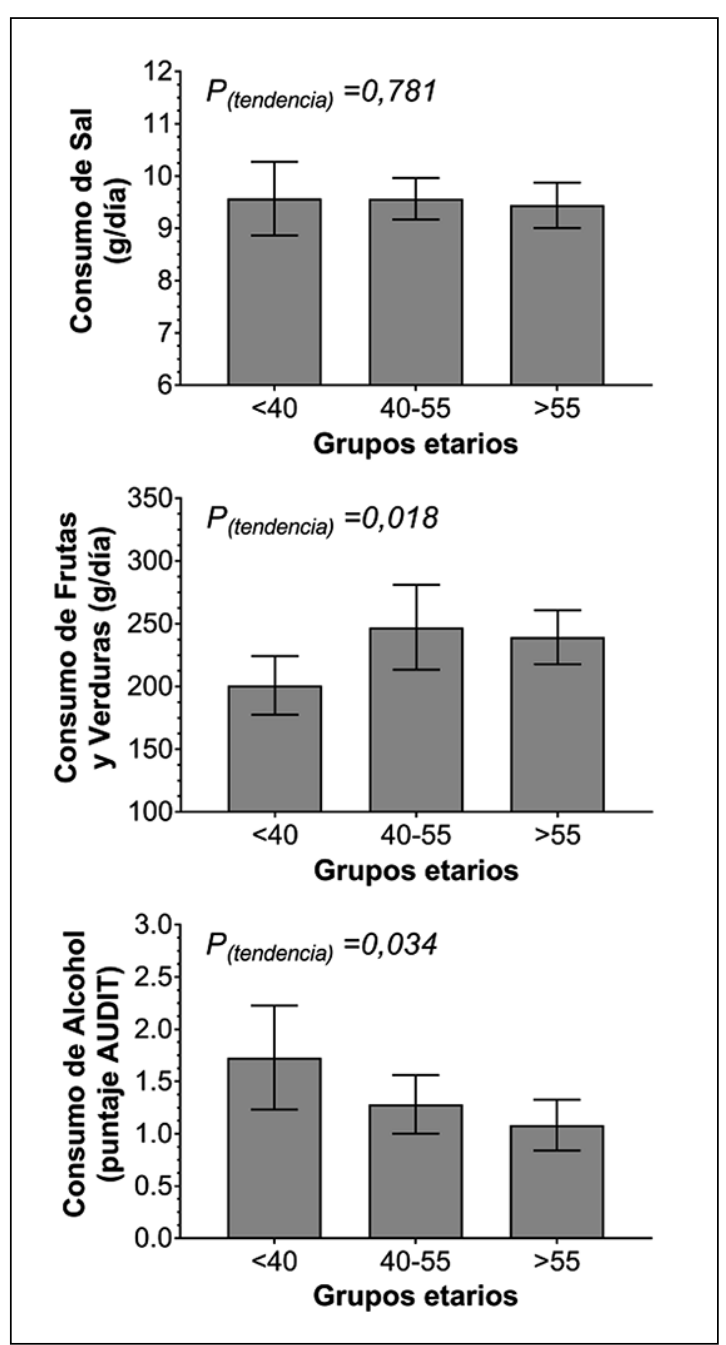

Figura 3. Consumo de frutas y verduras, sal y alcohol en dueñas de casa según grupo etario. Datos presentados como promedio y sus respectivos $95 \%$ IC, ajustados por nivel educacional y zona geográfica de residencia. El valor P-tendencia indica si el promedio de la variable de salida (consumo de sal, frutas y verduras y alcohol) presenta una tendencia a ser menor o mayor en la medida que la edad aumenta.

sin embargo, también se observó una mayor prevalencia de inactividad física en el grupo de dueñas de casa con mayor edad ( $>55$ años) en relación con los grupos más jóvenes. Esto concuerda con estudios previos, donde se ha reportado que dueñas de casa realizan menores niveles de AF en comparación a las mujeres que poseen un empleo (OR: 1,71 [95\% IC: 1,03 a 2,86], $\mathrm{p}=0,038)^{17}$.

Considerando los grandes beneficios que apor- ta la $\mathrm{AF}$, es pertinente fomentar hábitos de $\mathrm{AF}$ en este grupo de la población a través de programas específicos que podrían repercutir positivamente sobre su peso corporal y presión arterial, tal como ha sido demostrado previamente $e^{26,27}$. En el aspecto relacionado al estilo y calidad de vida en esta población, se ha observado una moderada-alta valoración por parte de las dueñas de casa ${ }^{28}$. Iniciativas, como las realizadas en México, como promover la educación, la igualdad de género, el crecimiento económico y del empleo, buscan garantizar el bienestar y la vida sana de toda su población, pero en particular de este grupo ${ }^{29}$.

\section{Fortalezas y limitaciones}

Dentro de las fortalezas de este estudio está la representatividad nacional de los datos utilizados, los cuales fueron corregidos mediante la aplicación de factores de expansión derivados por la ENS 2009-2010. Esta evidencia contribuye a aumentar la limitada evidencia científica en este grupo social. Otra fortaleza del estudio es la medición de estilos de vida mediante la utilización de instrumentos estandarizados. También cabe destacar que la presentación de los resultados estratificados por edad permite tener una aproximación al cumplimiento de los estilos de vida en dueñas de casa pertenecientes a diferentes generaciones, lo cual es relevante si consideramos que el aumento de actividad laboral renumerada en mujeres ha aumentado considerablemente durante las últimas dos décadas.

Este estudio también presenta limitaciones que tienen que ser consideradas al momento de interpretar los principales resultados de este trabajo. Una de ellas tiene relación a su naturaleza, ya que al ser un estudio de corte transversal no podemos establecer causalidad entre las variables de exposición y las variables de respuesta. Otra limitante tiene relación a los instrumentos de autorreporte utilizados para recolectar la información relacionada a estilos de vida de la ENS 20092010, los cuales están sujetos a un mayor grado de imprecisión y que tienen una mayor probabilidad de sobreestimar conductas saludables.

En base a los resultados de este estudio podemos concluir que las dueñas de casa $>55$ años presentan una mayor asociación de cumplir con al menos 4 de 7 estilos de vida saludable que dueñas de casa $<40$ años. Sin embargo, hay componentes específicos de los estilos de vida como lo son los 


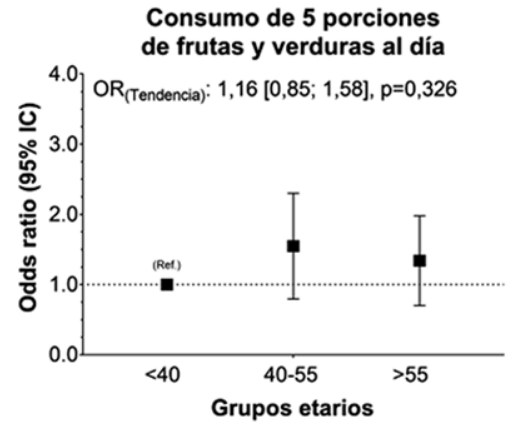

Puntaje AUDIT de consumo de alcohol $<8$

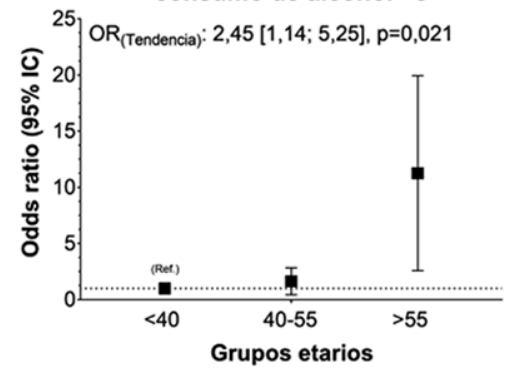

Horas de sueño 7-9 horas al día

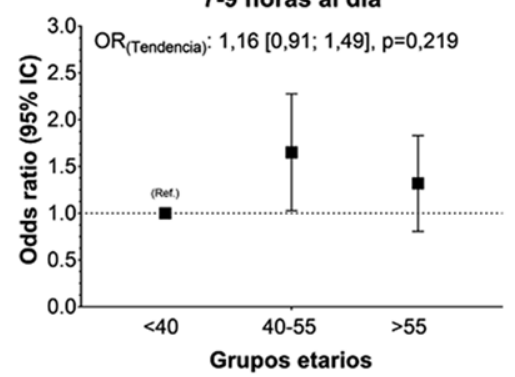

Físicamente activo (>150 minutos a la semana)

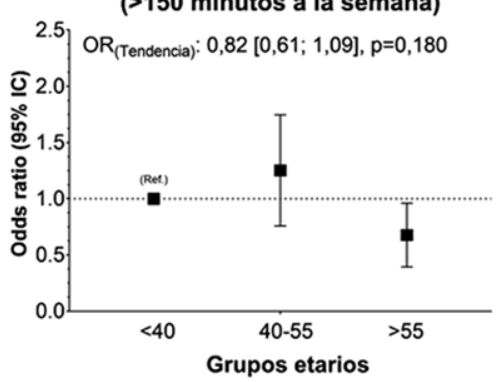

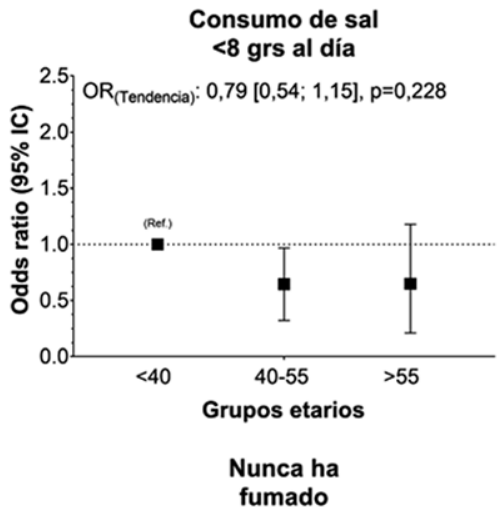
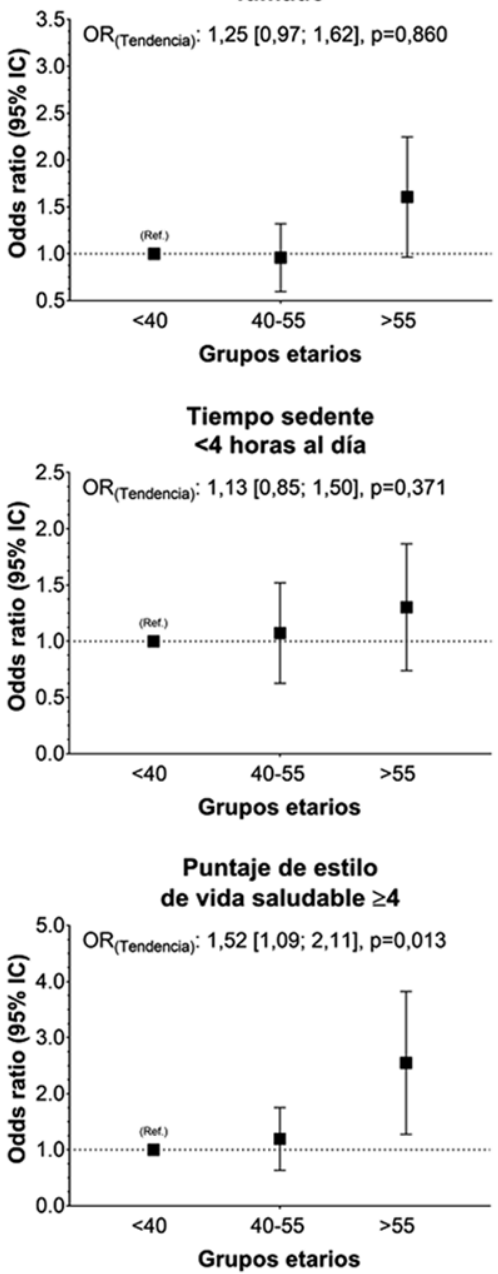

Figura 4. Cumplimiento de estilos de vida saludable en dueñas de casa según grupo etario. Datos presentados como OR y sus respectivos IC [OR (95\% IC)], ajustados por educación y zona geográfica de residencia. Se utilizó el grupo menor a 40 años como categoría de referencia para los análisis. El trend OR indica en cuánto se modifica el OR al desplazarse a la siguiente categoría de edad, un OR $<1$ indica que no cumplen con la recomendación de estilos de vida saludable, mientras que un OR $>1$ indica que cumplen con la recomendación. 
niveles de AF, consumo de frutas y verduras, y horas de sueño que aún podrían mejorarse mediante la creación de políticas públicas que incentiven la adopción de conductas y estilo de vida saludable en este grupo social.

Agradecimientos: Se agradece de manera especial a todos los participantes de la ENS 2009-2010, al equipo profesional de la Departamento de Salud Pública de la Facultad de Medicina de la Pontificia Universidad Católica de Chile, quienes desarrollaron y aplicaron la Encuesta Nacional de Salud y al Ministerio de Salud del Gobierno de Chile.

\section{Referencias}

1. Ministerio de Planificación. Estudio: transformaciones en las estructuras familiares en Chile; 2011. Disponible en: http://www.ministeriodesarrollosocial.gob.cl/btca/ txtcompleto/mideplan/transformac.fam.chilenas.pdf.

2. Ministerio de Desarrollo Social. Encuesta CASEN. Síntesis de resultados. Equidad de Género; 2015. Disponible en: http://observatorio.ministeriodesarrollosocial.gob.cl/ casen-multidimensional/casen/docs/CASEN_2015_Resultados_equidad_genero.pdf

3. Torres C. El trabajo doméstico y las amas de casa: El rostro invisible de las mujeres. Sociológica 1989; 4 (10).

4. Instituto Nacional de Estadísticas. Género y empleo. Informe; 2016. Disponible en: http://historico.ine.cl/ genero/files/estadisticas/pdf/documentos/enfoque-estadistico-genero-y-empleo.pdf.

5. Banco Mundial. Informe sobre el desarrollo mundial. Igualdad de género y desarrollo; 2012. Disponible en: https:// siteresources.worldbank.org/INTWDR2012/Resources/7778105-1299699968583/7786210-1315936231894/ Overview-Spanish.pdf.

6. Programa de las Naciones Unidas para el Desarrollo - PNUD. Informe sobre Desarrollo Humano; 2016. Disponible en http://hdr.undp.org/sites/default/files/ hdr_2016_report_spanish_web.pdf.

7. Ndungi FN, Tuitoek PJ, Aboud AA. Socio-economic status, knowledge, awareness and attitudes of the Swahili community in relation to dietary habits, obesity and lifestyle diseases. Afr J Food Agric Nutr Dev 2017; 17 (1): 11709-25.

8. Sadeghi M, Aghdak P, Motamedi N, Tavassoli A, Kelishadi R, Sarrafzadegan N. Do intervention strategies of Women Healthy Heart Project (WHHP) impact on differently on working and housewives? ARYA Atheroscler 2011; 6 (4): 129-35.
9. Demiral Y, Arik H, Toğrul BU. The association between employment status and metabolic syndrome in women: modifying effect of education. Women Health 2012; 52 (8): 755-70.

10. Jeong S, Cho SI. Concordance in the health behaviors of couples by age: A cross-sectional study. J Prev Med Public Health 2018; 51(1): 6-14.

11. Ministerio de Salud. Chile: Encuesta Nacional de Salud 2009-2010. Ministerio de Salud de Chile; 2010. Disponible en http://web.minsal.cl/portal/url/item/bcb03d7bc28b64dfe040010165012d23.pdf.

12. World Health Organization. Obesity: Preventing and Managing the Global Epidemic; 2000. Disponible en http://www.who.int/nutrition/publications/obesity/ WHO_TRS_894/en/

13. Organización Panamericana de la Salud. Parte 1: Módulos de Valoración Clínica. Módulo 5 Valoración Nutricional del Adulto Mayor; 2003. Disponible en: http:// www.sld.cu/galerias/pdf/sitios/gericuba/modulo5.pdf.

14. World Health Organization. Global Physical Activity Questionnaire: GPAQ version 2.0. World Health Organization; 2009. Disponible en: http://www.who.int/chp/ steps/resources/GPAQ_Analysis_Guide.pdf.

15. Celis-Morales C, Salas C, Alduhishy A, Sanzana R, Martinez MA, Leiva A, et al. Socio-demographic patterns of physical activity and sedentary behaviour in Chile: results from the National Health Survey 2009-2010. J Public Health 2015; 38 (2): 98-105.

16. Babor TF, Higgins-Biddle JC, Saunders JB, Monteiro MG. The alcohol use disorders identification test. Guidelines for Use in Primary Care, 2; 2001. Disponible en: http://apps.who.int/iris/bitstream/handle/10665/67205/ WHO_MSD_MSB_01.6a.pdf;jsessionid=6A0A6A6BD1C8DC1C50FF8B32A9F2F294? sequence $=1$.

17. Jalali-Farahani S, Amiri P, Bakht S, Shayeghian Z, Cheraghi L, Azizi F. Socio-demographic determinants of health-related quality of life in Tehran lipid and glucose study (TLGS). Int J Endocrinol Metab 2017; 15 (4): e14584.

18. Baheiraei A, Bakouei F, Mohammadi E, Montazeri A, Hosseni M. The social determinants of health in association with women's health status of reproductive age: A population-based study. Iran J Public Health 2015; 44 (1): 119-29.

19. Córdoba L, González MR, Obando D, Coulacoglou C. Current Trends in Family Structures in Four Colombian Cities. Population Review 2013; 52 (2): 119-34.

20. Morales A, Pozo C, Quilodrán J, Moglia S, Kaempffer A. Perfil sociodemográfico y de riesgo cardiovascular en pacientes diabéticos de un consultorio urbano SSMN, 2004. Rev Chil Salud Pública 2006; 10 (3): 130-8. 
21. Dois A, Cazenave A. Pesquisa de depresión mayor en hipertensos consultantes de la atención primaria. Rev Med Chile 2009; 137 (4): 475-80.

22. Cid P, Merino JM, Stiepovich J. Factores biológicos y psicosociales predictores del estilo de vida promotor de salud. Rev Med Chile 2006; 134 (12): 1491-99.

23. Vidal Gutiérrez D, Chamblas García I, Zavala Gutiérrez M, Müller Gilchrist RALPH, Rodríguez Torres MC, et al. Determinantes sociales en salud y estilos de vida en población adulta de Concepción, Chile. Cienc Enferm 2014; 20 (1): 61-74.

24. Lee T, Khor W, Tan N, Cheng C, Seow A, Foo S. A cross-sectional survey of physical activity among middle aged women in Singapore. Singapore Med J 1999; 40 (7): 468-76.

25. Tarducci G, Barengo N, Morea G, Gárgano S, Gandini A, Paganini A, et al. Relación entre el nivel de escolaridad y el patrón de actividad física en Balcarce, Argentina. Hacia Promoc Salud 2016; 21 (2): 89-98.
26. Kassim MSA, Manaf MRA, Nor NSM, Ambak R. Effects of lifestyle intervention towards obesity and blood pressure among housewives in Klang valley: a quasi-experimental study. Malays J Med Sci 2017; 24 (6): 83-91.

27. Mohamad Nor N, Ambak R, Omar MA, Shahar S, Abdul Aziz NS, et al. Methodology of the my body is fit and fabulous at home (MyBFF@ home): an intervention study to combat obesity among housewives in Malaysia. J Womens Health 2016; 5 (5): 2-8.

28. Staland-Nyman C, Alexanderson K, Hensing G. Associations between strain in domestic work and self-rated health: a study of employed women in Sweden. Scand J Public Health 2008; 36 (1): 21-7.

29. De Castro F, Rojas R, Villalobos A, Allen B, Hubert C, Romero $\mathrm{M}$, et al. Bases metodológicas y resultados de la implementación de la Encuesta Nacional de Niños, Niñas y Mujeres en México 2015. Salud Publ Mex 2015; 58 (6): 676-84. 